(2) Open Access Full Text Article

\title{
Preparation of novel biodegradable ropivacaine microspheres and evaluation of their efficacy in sciatic nerve block in mice
}

This article was published in the following Dove Press journal:

Drug Design, Development and Therapy

4 August 2016

Number of times this article has been viewed

\author{
Qiang Ni \\ Wurong Chen \\ Lei Tong \\ Jue Cao \\ Chao Ji
}

Department of Anesthesiology, Putuo Hospital, Shanghai University of Traditional Chinese Medicine, Shanghai, People's Republic of China

Correspondence: Wurong Chen Department of Anesthesiology, Putuo Hospital, Shanghai University of Traditional Chinese Medicine, 164 LanXi Road, Putuo District, Shanghai 200062,

People's Republic of China

Tel/fax +86 2l 22233222

Email 2472336724@qq.com

\begin{abstract}
In this study, ropivacaine chitosan-loaded microspheres for subcutaneous administration were developed. The systems were characterized in terms of surface morphology, particle size, encapsulation efficiency, and in vitro release behavior. Results showed that the microspheres had drug loading rate of $7.3 \%$ and encapsulation efficiency of $91.2 \%$, and their average diameter was $2.62 \pm 0.76 \mu \mathrm{m}$. The morphology study revealed that the microspheres are uniform monodispersed spheres and did not form aggregates in aqueous solution. It was clearly observed that the release profile of ropivacaine microspheres exhibited a biphasic pattern: the initial burst release within the first 2 hours and a following slower and sustained release over a long time. In vivo, a greater area under the plasma concentration-time curve from 0 to $t\left(\mathrm{AUC}_{0-t}\right)$ was obtained from the microspheres (4.27-fold), than from the injection group, which indicated that there was a significantly improved systemic exposure to ropivacaine. Pharmacodynamics result showed that preparing ropivacaine as microsphere preparation could not only extend the drug effect time but also decrease the administration dosage.
\end{abstract}

Keywords: ropivacaine, chitosan, microspheres, in vitro release, pharmacodynamics

\section{Introduction}

Local anesthetics are widely used in postoperative analgesia, obstetric and dental pain, as well as in the therapy of chronic pain. ${ }^{1}$ Among these drugs, ropivacaine (RPC) plays a valuable role in the overall management of surgical and postoperative pain. $\mathrm{RPC}$ is a long-acting local anesthetic and is a pure enantiomeric isomer(s) amide similar to bupivacaine (Figure 1). ${ }^{2}$ However, it may be a priority option because of its reduced potential central nervous system (CNS) and cardiotoxicity, as well as its lower propensity for motor block. ${ }^{3}$ The higher $\mathrm{pKa}$ and lower lipid solubility of RPC enable blocking of nerve fibers involved in pain transmission (A delta and $\mathrm{C}$ fibers) to a greater extent than those controlling motor function (A beta fibers). The drug is less toxic than the same concentration of bupivacaine but more toxic than lignocaine; it has a significantly higher threshold for CNS toxicity than bupivacaine. A large number of clinical data indicate that epidural RPC $0.2 \%$ is effective in the initiation and maintenance of labor analgesia and provides pain relief after abdominal or orthopedic surgery, especially when given in association with opioids. ${ }^{3-5}$

Local anesthetics can temporarily and reversibly block local nerve conduction and thus lead to the analgesic effect. However, clinically, water-soluble local anesthetics cannot achieve long-time postoperative analgesia by single injection, with normal analgesia period not being longer than 12 hours. ${ }^{6}$ To meet the long-time analgesia requirement in the clinic, methods such as discontinuous injection of anesthetic or (c)
hereby accept the Terms. Non-commercial uses of the work are permitted without any further permission from Dove Medical Press Limited, provided the work is properly attributed. For permission for commercial use of this work, please see paragraphs 4.2 and 5 of our Terms (https://www.dovepress.com/terms.php). 
A

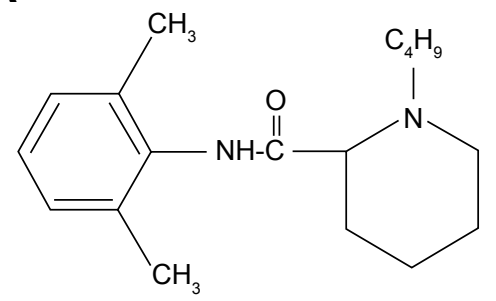

B

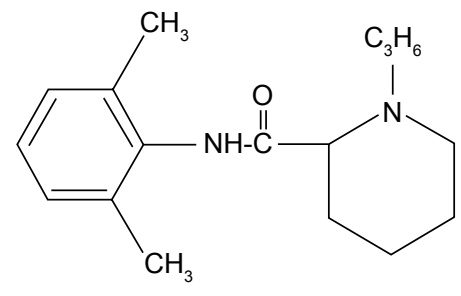

Figure I Structure of $(\mathbf{A})$ bupivacaine and $(\mathbf{B})$ ropivacaine.

catheter implantation in vivo and continuation of medication to achieve the desired effect have been applied. However, it requires relatively expensive equipment and continuous monitoring; long-time retention of catheter could easily cause infection and catheter displacement. Thus, it was not the best analgesia method and clinical settings required discovery of a better method. ${ }^{7,8}$

Microspheres have been explored extensively for their use in the field of drug delivery, and various polymers have been utilized for the formulation of the microspheres, which in turn have been assessed for different purposes. ${ }^{9}$ This drug delivery system has become a therapeutic measure to improve the delivery of drugs to a considerable extent in a specific location and has already been applied to improve the treatment response and to reduce adverse effects. ${ }^{10,11}$

Eventually, the total dose and few adverse reactions may be reduced because a steady plasma concentration is maintained. Microspheres are potential drug delivery carrier systems in the segment of novel drug delivery and are prepared using assorted polymers. ${ }^{12-14}$

Chitosan, which is the deacetylated derivative of $\beta(1 \rightarrow 4)$ 2-acetamido-2-deoxy-D-glucose or chitin, has been extensively explored for its various biomedical and pharmaceutical applications. Properties such as biodegradability, low toxicity, and good biocompatibility make it suitable for use in drug delivery and in the biomedical field. ${ }^{15,16}$ As a drug carrier, chitosan has been investigated for the sustained delivery of many oral formulations and parenteral formulations ${ }^{17}$ Chitosan microspheres have been prepared by emulsion crosslinking, ion-induced coagulation, and spray-drying methods. Of these methods, the most common method used to prepare chitosan microspheres is the emulsion cross-linking method. ${ }^{18}$ There are numerous reports on the use of glutaraldehyde as a cross-linking agent in the preparation of microspheres. ${ }^{19,20}$ The chemical cross-linking method for preparation of chitosan microspheres involves emulsification, followed by crosslinking with a suitable cross-linking agent.

In this study, we developed chitosan-loaded microspheres for subcutaneous administration of RPC in order to achieve a controlled drug release profile. Polymeric microspheres were prepared by an "emulsion cross-linking method" using chitosan as matrix. The systems were characterized in terms of surface morphology and size distribution. Technological studies were performed to evaluate the drug particle size, encapsulation efficiency, and its in vitro release behavior. In addition, the pharmacokinetics and pharmacodynamics of RPC-loaded chitosan microspheres were investigated.

\section{Materials and methods Materials}

RPC hydrochloride was a gift from Belka Biopharm (Wuhan, Hubei, People's Republic of China). Ultrapure biomedical grade chitosan $(>74.5 \%$ deacetylation) was obtained from Dixin Chemical (Wuhan, Hubei, People's Republic of China). Glutaraldehyde, liquid paraffin (analytical grade), Span 80, mannitol, Tween 20, and phosphate-buffered saline (PBS) were purchased from Sinopharm (Shanghai, People's Republic of China). All other materials or solvents were of reagent or analytical grade. Deionized distilled water was generated by a Millipore Milli-Q integral water purification system (Shanghai, People's Republic of China).

\section{Preparation of microspheres}

The mucoadhesive microspheres containing RPC were prepared using the water-in-oil emulsion cross-linking method. Chitosan (100 mg) was dissolved in 1\% acetic acid solution. The drug (40 mg) was dispersed in the polymer solution as a water phase. The chitosan solution was added into the oil phase of liquid paraffin $(25 \mathrm{~mL})$ containing $1 \%$ Span 80 as an emulsifying agent with constant stirring using a stirrer. Then, 20\% glutaraldehyde solution was slowly added to the emulsion system and cross-linked for 4 hours until the microspheres were solidified. The microspheres obtained were separated by centrifugation and washed with isopropyl alcohol to remove liquid paraffin. After addition of $1 \mathrm{~mL}$ of aqueous mannitol $(20 \%, \mathrm{w} / \mathrm{v})$ to prevent the aggregation of microparticles, the microspheres were freeze-dried. 


\section{Characterization}

The morphology of RPC-loaded chitosan microspheres was studied by scanning electron microscopy (SEM) (JSM-6400 electron microscope, JEOL, Tokyo, Japan). The microspheres were fixed on a rigid support and coated with gold. The average particle size of the prepared RPC-loaded microspheres was determined by dynamic light scattering system (Malvern Instruments, Malvern, UK) using a NICOMP 380 Micron Particle Sizer (Particle Sizing Systems, Santa Barbara, CA, USA). Sample solutions were transferred to the light-scattering cells. At room temperature, the intensity autocorrelation was measured at a scattering angle of $90^{\circ}$. Data were analyzed in terms of intensity-weighted NICOMP distributions (PSS, Port Richey, FL, USA). The experimental results for each report are the average of at least three values obtained from analysis of the autocorrelation function accumulated for at least 20 minutes. Zeta potential was measured on the same samples prepared for size analysis.

\section{Drug content, entrapment efficiency, and drug loading}

The drug content of RPC microspheres was determined for various batches of the microspheres. Briefly, the powdered microspheres were dissolved in adequate quantity of PBS and then filtered. The drug content was detected by highperformance liquid chromatography (HPLC). The drug entrapment efficiency and drug loading were calculated using the following equations, respectively:

$$
\begin{aligned}
& \mathrm{EE}(\%)=\frac{M_{1}-M_{2}}{M_{1}} \times 100 \% \\
& \operatorname{DL}(\%)=\frac{W_{\mathrm{RPC}}}{W_{\text {microspheres }}} \times 100 \%
\end{aligned}
$$

$W_{\mathrm{RPC}}$ represents the amount of RPC loaded in the microspheres, $W_{\text {microspheres }}$ represents the weight of the RPC microspheres, and $M_{1}$ and $M_{2}$ are defined as the masses of the initially added RPC and the nonencapsulated RPC, respectively.

\section{In vitro release}

Release studies were performed with a modified dialysis bag method ${ }^{21}$ using a rotating paddle apparatus. The release medium was PBS $(890 \mathrm{~mL}, \mathrm{pH} 7.4)$, thermostated at $37^{\circ} \mathrm{C}$ and meeting the sink conditions. Weighted amounts of RPC-loaded microspheres ( $25 \mathrm{mg})$ were suspended in $10 \mathrm{~mL}$ of aqueous solution containing $0.05 \%$ Tween 20 and then dropped in the release medium. Samples of $2 \mathrm{~mL}$ were removed at sampling times of $0.5,1,2,4,6,8,10,12$ and 24 hours after centrifugation at $4,000 \mathrm{rpm}$ for 5 minutes. The medium removed from the tubes was replaced with the same amount of fresh release medium. The collected supernatants $(20 \mu \mathrm{L})$ were analyzed for RPC content by reversed-phase (RP)-HPLC. Each batch of microspheres was analyzed six times. RPC injection was used for the control group.

\section{Animals}

The experiments were performed on Sprague Dawley rats $(220 \pm 20 \mathrm{~g})$ and Kunming mice $(20 \pm 2 \mathrm{~g})$. Animals were maintained in a temperature- and humidity-controlled vivarium, on a 12-hour/12-hour light/dark cycle, with access to rodent chow and water ad libitum. All animal study protocols were approved by the Institutional Animal Care and Use Committee at the Hospital of Shanghai, and were in accordance with the Guide for the Care and Use of Laboratory Animals.

\section{Pharmacokinetic evaluation}

Twelve rats were used to investigate the effect of microsphere formulation on the pharmacokinetics of RPC after subcutaneous administration. Rats were divided into two groups at random and given a single $10 \mathrm{mg} / \mathrm{kg}$ dose of the RPC-loaded microspheres or RPC injection by subcutaneous injection. Blood samples $(0.5 \mathrm{~mL})$ were collected into heparinized tubes from the caudal vein at $0.5,1,2,4,6,12,24,36,48$, 72 , and 96 hours after subcutaneous administration. Blood was immediately processed for plasma by centrifugation at $3,500 \times g$ for 10 minutes. Plasma samples were frozen and maintained at $-70^{\circ} \mathrm{C}$ until analysis.

Pharmacokinetic parameters were calculated against the plasma concentration-time data. The maximum observed concentration $\left(C_{\max }\right)$ and time to $C_{\max }\left(T_{\max }\right)$ were directly calculated from the plasma concentration vs time curve. The elimination rate constant $\left(K_{\mathrm{el}}\right)$ was determined from the terminal stage of the log plasma concentration vs time curve by least-squares regression analysis. The $K_{\mathrm{el}}$ is calculated as $K_{\mathrm{el}}=$ slope $\times 2.303$. The elimination half-life is calculated as $t_{1 / 2}=0.693 / K_{\mathrm{el}}$. The area under the plasma concentration-time curve from 0 to $t\left(\mathrm{AUC}_{0-t}\right)$ and from 0 to $\infty\left(\mathrm{AUC}_{0-\infty}\right)$ was calculated using the trapezoidal rule.

\section{HPLC analysis}

The analysis of RPC levels in vitro and in vivo were carried out using RP-HPLC on a system equipped with an Agilent 1100 Series and a HS2000 interface (Agilent Technologies, Santa Clara, CA, USA) operated at $262 \mathrm{~nm}$. The column was a Diamonsil $\mathrm{C}_{18}$ HPLC column (Dikma Technologies Inc, Lake Forest, CA, USA; $5 \mu \mathrm{m}, 200 \times 4.6 \mathrm{~mm}$ ). The mobile 
phase consisted of 50\% PBS and 50\% acetonitrile (containing $0.1 \%$ trifluoroacetic acid), and the flow rate was $1.0 \mathrm{~mL} / \mathrm{min}$. The column temperature was $30^{\circ} \mathrm{C}$. A $100 \mu \mathrm{L}$ volume of the plasma sample was transferred to a $5 \mathrm{~mL}$ plastic test tube together with $10 \mu \mathrm{L}$ of internal standard solution $(10 \mu \mathrm{g} / \mathrm{mL})$. After vortex shaking for 1 minute, $300 \mu \mathrm{L}$ of acetonitrile was added for precipitation. After centrifugation at $3500 \times g$ for 10 minutes, the clear supernatant was removed, and $20 \mu \mathrm{L}$ of the sample was taken for HPLC analysis. Dexamethasone was used as internal standard in this study.

\section{Pharmacodynamic evaluation}

The experimental mice were placed on a hot pedal preheated to $51^{\circ} \mathrm{C}$, with temperature range of $50^{\circ} \mathrm{C} \pm 0.5^{\circ} \mathrm{C}$. The latent period of mice licking their posterior paws was used as the pain threshold indicator. Each mouse was evaluated three times, with interval period of 5 minutes. The average value of the three measurements was used as the basic pain threshold. Animals with response latency $<5$ seconds or $>30$ seconds were eliminated. ${ }^{22}$

Kunming mice $(n=60)$ were randomly divided into five groups after screening: blank microspheres group (A), RPC injection group (B, $400 \mathrm{mg} / \mathrm{kg}), \mathrm{RPC}$ microspheres low-dosage group (C, $200 \mathrm{mg} / \mathrm{kg})$, RPC microspheres mediumdosage group (D, $400 \mathrm{mg} / \mathrm{kg}$ ), RPC microspheres highdosage group (E, $600 \mathrm{mg} / \mathrm{kg}$ ). The mice had access to food 12 hours before administration of the injections and were free to drink water. Next, $2 \%$ sevoflurane was used for anesthesia, and then surgery with sciatic nerve embedding administration of medicine was applied. After anesthesia, mice were laid on a panel, and the hip hair was shaved (under the ischium: $0.3-0.5 \mathrm{~cm}$ ), and the skin under and parallel with the ischium was cut; a small hemostat or small scissors was used to passively fully expose the sciatic nerve. Each group was injected with blank microspheres, RPC injection, and RPC microspheres in the muscle gap around the sciatic nerve. To prevent mice from disturbing the wound stitches by themselves or others, absorbable suture was used to sew muscle and skin. ${ }^{23,24}$

After administration of each formulation to the different groups at $0.5,1,2,4,8,16,24,36,48,72$, and 96 hours, the hot pedal method was used to measure the latency of mice licking the side of the posterior paws injected with microspheres after a thermal stimulus. Twelve mice were evaluated for each group at each time point to avoid heat-variable reaction after several hot pedal thermal stimuli, which may lead to data distortion. Each measurement for each mouse had an interval of at least 30 minutes. The latent time of licking of posterior paws was expressed as mean \pm standard deviation (SD). If the mice did not have reaction of pulling back paws or of licking posterior paws within 30 seconds, to avoid causing tissue damage, the measured posterior paws were removed from the hot pedal, and the latent time was marked as 30 seconds.

\section{Statistical analysis}

Results are expressed as mean \pm SD of three experiments. Data analysis of the pharmacokinetic parameters was performed using the unpaired Student's $t$-test. A value of $P<0.05$ was considered significant.

\section{Results and discussion Characterization}

In this study, emulsion cross-linking method was used to prepare the RPC-loaded chitosan microspheres. RPC and chitosan were first dissolved in $1 \%$ acetic acid solution and emulsified in a solution of liquid paraffin to form the primary emulsion. After cross-linking for 4 hours, the microspheres were solidified and freeze-dried. The microspheres had drug loading of $7.3 \%$ and encapsulation efficiency of $91.2 \%$. The diameter of RPC-loaded chitosan microspheres was in the range of $0.72-5.16 \mu \mathrm{m}$. The size of $22 \%$ of them was between $0.72 \mu \mathrm{m}$ and $1.24 \mu \mathrm{m} ; 56 \%$ of microspheres were of sizes between $1.39 \mu \mathrm{m}$ and $2.97 \mu \mathrm{m}$, and $22 \%$ of microspheres were between $3.04 \mu \mathrm{m}$ and $5.16 \mu \mathrm{m}$. The average diameter of RPC-loaded chitosan microspheres was $2.62 \pm 0.76 \mu \mathrm{m}$. The morphology of the RPC-loaded chitosan microspheres observed by SEM is shown in Figure 2, which reveals that

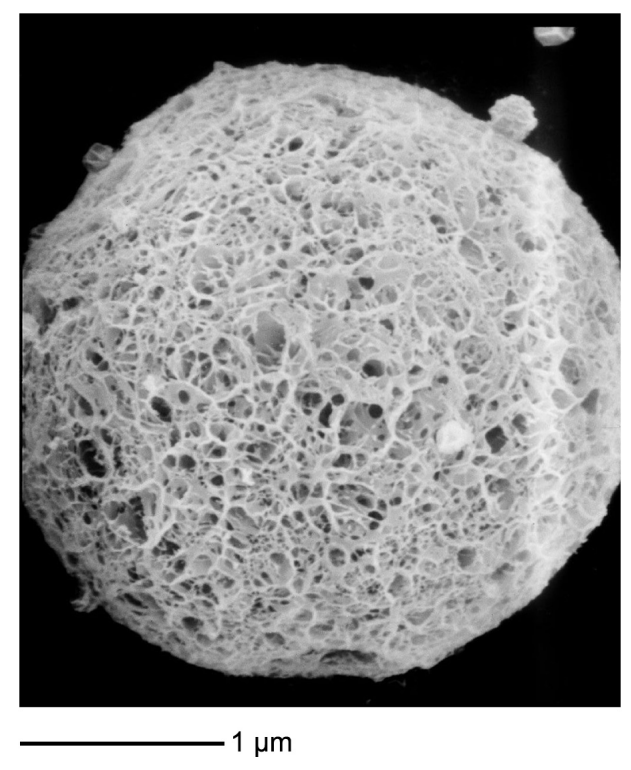

Figure 2 The morphology of the RPC-loaded chitosan microspheres observed by SEM.

Abbreviations: RPC, ropivacaine; SEM, scanning electron microscopy. 


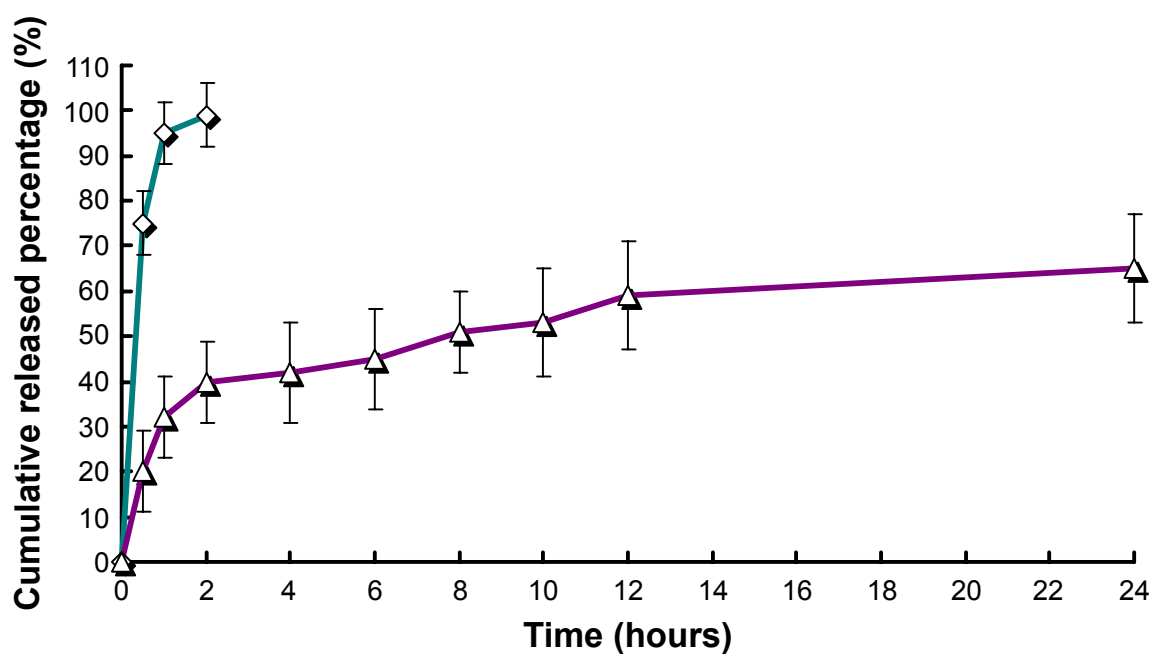

Figure 3 The release profiles of free RPC $(\diamond$, jade line) and RPC-loaded chitosan microspheres $(\Delta$, purple line) $(n=3)$.

Note: Each point represents the mean \pm standard deviation.

Abbreviation: RPC, ropivacaine.

the self-assembled microspheres are uniform monodispersed spheres and did not form aggregates in aqueous solution.

\section{Analysis method validation}

The linear range of the assay for the plasma of mice was $5-1,000 \mathrm{ng} / \mathrm{mL}$, and the standard curve of RPC was $Y=13.27 X+0.251 \quad(r=0.9994)$. The intra- and interday assay variability for all samples did not exceed $8.5 \%$ and $12.7 \%$, respectively. The method recoveries were in the range of $88 \%-105 \%$. The detection limits and quantitation limits of liquid chromatography analysis were respectively determined to be $5 \mathrm{ng} / \mathrm{mL}$ for the in vivo plasma samples. Moreover, there were no interfering peaks observed in all the chromatograms. It was noteworthy that the HPLC technique, although simple, was an effective method to analyze the RPC-in-microspheres system.

\section{In vitro release}

The release profile of free RPC and RPC-loaded chitosan microspheres is shown in Figure 3. In comparison to free RPC, the release behavior of RPC microspheres exhibited a sustained manner. It was clearly observed that the release profile of RPC microspheres exhibited biphasic patterns: the initial rapid release within the first 2 hours and a following slower and sustained release over a long time.

The release of RPC from microspheres was controlled during the first stages by diffusion due to the small molecular weight of this drug; thus, the drug placed on the most superficial part of the microspheres was released quickly, where the contribution of the smaller microspheres was bigger. In contrast, $>80 \%$ of RPC was released in the first hour of the dissolution process into the release medium. Almost $100 \%$ of RPC was released in the first 2 hours. To predict the release kinetics, several drug release models (including firstorder, Higuchi, Korsmeyer-Peppas, and Weibull distribution models) were adopted, and the results are shown in Table 1.

The RPC release profile from the microspheres was best fitted with the Weibull distribution model $\left(r^{2}=0.9931\right)$. The result of the release study indicated that the mechanism of drug release from microspheres was the coalition of diffusion and erosion.

\section{Pharmacokinetic studies}

The plasma concentration-time profiles of RPC after subcutaneous injection of the RPC microspheres and injection in rats are shown in Figure 4. In general, the maximum plasma

Table I Release kinetics of RPC-loaded microspheres

\begin{tabular}{|c|c|c|c|c|}
\hline \multirow[t]{2}{*}{ Model } & \multicolumn{2}{|l|}{ Free RPC } & \multicolumn{2}{|l|}{ RPC-loaded microspheres } \\
\hline & Equation & $\begin{array}{l}\text { Correlation } \\
\text { coefficient }\left(r^{2}\right)\end{array}$ & Equation & $\begin{array}{l}\text { Correlation } \\
\text { coefficient }\left(r^{2}\right)\end{array}$ \\
\hline First order & $\ln (I-Q)=3.276 t-1.092$ & 0.9432 & $\ln (I-Q)=5.276 t+0.562$ & 0.9534 \\
\hline Higuchi & $Q=8.211 t_{1 / 2}+1.219$ & 0.9821 & $Q=8.627 t_{1 / 2}+1.921$ & 0.9812 \\
\hline Korsmeyer-Peppas & $\ln Q=0.256 \ln t-0.627$ & 0.9525 & $\ln Q=1.287 \ln t+0.192$ & 0.9546 \\
\hline Weibull & $\ln \ln (I /(I-Q))=3.29 I \ln t-1.28 I$ & 0.9829 & $\ln \ln (I /(I-Q))=I .982 \ln t+0.26 I$ & 0.9931 \\
\hline
\end{tabular}

Abbreviation: RPC, ropivacaine. 


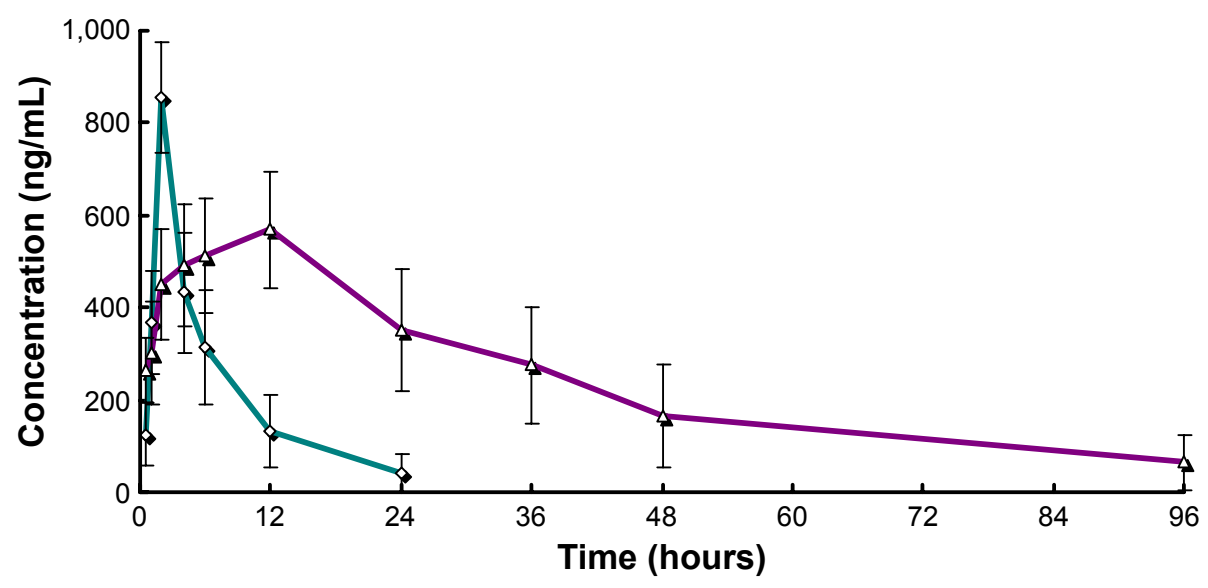

Figure 4 Mean plasma concentration-time profiles for free RPC $(\nabla$, jade) and RPC-loaded chitosan microspheres $(\Delta$, purple) administered to rats Note: Each point represents the mean \pm standard deviation of six rats.

Abbreviation: RPC, ropivacaine.

concentration of RPC microspheres was $569 \pm 126 \mathrm{ng} / \mathrm{mL}$, and it was reached 12 hours after subcutaneous injection. From this time, the plasma drug concentration gradually decreased and the drug was not detected after 96 hours. When the drug was administered in solution at the same dose by subcutaneous injection, the maximum drug concentration was obtained at 2 hours, and the drug was detected in the plasma only for 24 hours. The pharmacokinetic parameters of RPC in the formulations are shown in Table 2. As shown in Table 2, the half-life of RPC injection (1.79 hours) was shorter than that of RPC microspheres (15.62 hours), suggesting that the injection was taken up by other tissues more rapidly than the microsphere formulation. A greater $\mathrm{AUC}_{0-t}$ was obtained with the microspheres (4.27-fold), than from the injection, which indicated that there was a significantly improved systemic exposure to RPC due to the sustained release of microspheres. The clearance of RPC microspheres was $1.21 \mathrm{~L} / \mathrm{h}$, smaller than that $(8.26 \mathrm{~L} / \mathrm{h})$ of the RPC injection.

\section{Pharmacodynamic evaluation}

This experiment used the mice hot pedal stimulation model to evaluate the pharmacodynamics of the RPC microspheres.

Table 2 Pharmacokinetic parameters of the two formulations

\begin{tabular}{lll}
\hline Parameter & \multicolumn{2}{l}{ Formulations } \\
\cline { 2 - 3 } & Injection & Microspheres \\
\hline$t_{1 / 2}$ (hours) & $1.79 \pm 0.65$ & $9.13 \pm 3.32$ \\
$C_{\max }(\mathrm{ng} / \mathrm{mL})$ & $856.5 \pm 121.4$ & $569.4 \pm 126.3 *$ \\
$\mathrm{AUC}_{0-t}(\mathrm{ng} \cdot \mathrm{h} / \mathrm{mL})$ & $5,439.6 \pm 541.7$ & $23,210.2 \pm 2,315.4^{*}$ \\
$\mathrm{AUC}_{0-\infty}(\mathrm{ng} \cdot \mathrm{h} / \mathrm{mL})$ & $5,864.9 \pm 598.2$ & $25,265.6 \pm 2,448.6 *$ \\
$\mathrm{CL}(\mathrm{L} / \mathrm{h})$ & $8.26 \pm 1.37$ & $2.14 \pm 0.7 \mathrm{I}$ \\
\hline
\end{tabular}

Note: $* P<0.05, \mathrm{RPC}$ injection vs microspheres.

Abbreviations: $\mathrm{AUC}$, area under the concentration-time curve; $\mathrm{CL}$, clearance; $C_{\max }$, maximum observed concentration; RPC, ropivacaine; $t_{1 / 2}$, elimination half-life.
After testing and eliminating unsuitable animals, the mice in the blank group had average response latency of $\sim 12$ seconds (Figure 5). In the experimental group, the latent period was significantly increased after injection of RPC, extending from 12 seconds to 30 seconds ( 1 hour), but the effect time could not be maintained for a long time. Around 2 hours after administration, the latent period after stimulation gradually reverted to the initial level. Eight hours after administration, the basic drug effect was fully released. The latent period for mice in the control group was the same (12 seconds). On the contrary, in the RPC microspheres group, all the three dosages showed the obvious effect of extending the latent period to various degrees, apart from showing dose dependency. In the high-dosage group, the latent period was extended beyond 2 hours of administration and was maintained at a relatively high level (35 seconds), with an obvious drug effect until 72 hours. In the low-dosage group, although the dosage was half that of the injection group, considering the pharmacodynamics result, overall the two groups had equivalent treatment effect with no significant difference. As is well known, RPC is a local anesthesia drug that is clinically applied for the treatment of acute and chronic pain. Compared to other caine drugs, RPC had appropriate effect time and relatively long action period. Its main features included separated movement of dosage relationship and block anesthesia, low toxicity in nervus centralis and angiocarpy, and other merits. However, its longest sensation of pain block effect time was $<12$ hours. For chronic pain patients, it still required multiple dosing or continuous dosing with long-period placement of catheter in vivo. It caused poor compliance in patients clinically and high cost. The pharmacodynamics result showed that preparing RPC as a microsphere preparation could not only 


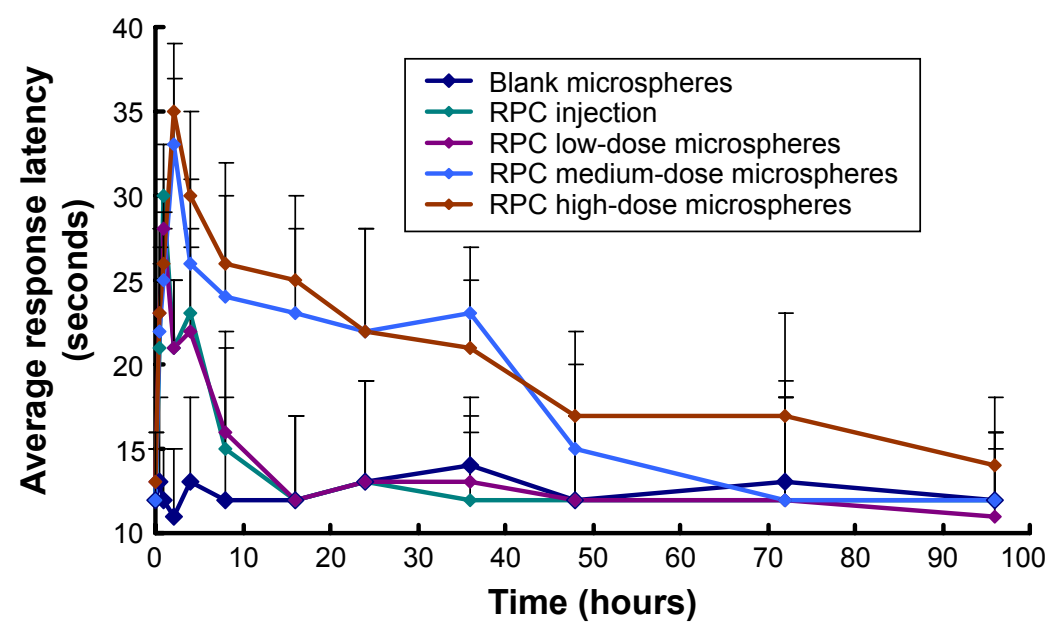

Figure 5 Pharmacodynamic evaluation of RPC in different formulations administered to mice. Note: Each point represents the mean \pm SD of 12 mice.

Abbreviations: RPC, ropivacaine; SD, standard deviation.

extend the drug effect time but also decrease the administration dosage.

\section{Conclusion}

In this study, RPC chitosan-loaded microspheres for subcutaneous administration were developed. The systems were characterized in terms of surface morphology, particle size, encapsulation efficiency, and in vitro release behavior. Results showed that the microspheres had drug loading of $7.3 \%$ and encapsulation efficiency of $91.2 \%$; the average diameter was $2.62 \pm 0.76 \mu \mathrm{m}$. The morphology study revealed that the microspheres are homogeneous monodisperse microspheres and did not form aggregates in aqueous solution. It was clearly observed that the release profile of RPC microspheres exhibited biphasic patterns: the initial rapid release within the first 2 hours, followed by a slower and sustained release over a long time.

In vivo, a greater $\mathrm{AUC}_{0-t}$ was obtained with the microspheres (4.27-fold), than in the injection group, which indicated that there was a significantly improved systemic exposure to RPC. The pharmacodynamics result showed that preparing RPC as a microsphere preparation could not only extend the drug effect time, but also decrease the administration dosage.

\section{Disclosure}

The authors report no conflicts of interest in this work.

\section{References}

1. Andreae MH, Andreae DA. Local anaesthetics and regional anaesthesia for preventing chronic pain after surgery. Cochrane Database Syst Rev. 2012;10:CD007105.
2. Zhai Y, Zhao L, Wang Z, Zhai G. Preparation and characterization of novel lipid nanocapsules of ropivacaine for transdermal delivery. Drug Deliv. 2016;23(2):619-628.

3. McClellan KJ, Faulds D. Ropivacaine: an update of its use in regional anaesthesia. Drugs. 2000;60(5):1065-1093.

4. Woloszczuk-Gebicka B, Grabowski T, Borucka B, Karas-Trzeciak M. Pharmacokinetics of sufentanil administered with $0.2 \%$ ropivacaine as a continuous epidural infusion for postoperative pain relief in infants. Paediatr Anaesth. 2014;24(9):962-967.

5. Lee JH, Kim NO, Ahn EK. A comparison of epidural infusion of 0.2 , 0.25 , and $0.3 \%$ ropivacaine with fentanyl after unilateral total knee arthroplasty. Korean J Anesthesiol. 2013;65(2):180-181.

6. Shipton EA. New formulations of local anaesthetics-part I. Anesthesiol Res Pract. 2012;2012:546409.

7. Thomas F, Drolet P, Varin F. Simultaneous percutaneous implantation of a microdialysis probe for monitoring perineural concentrations of local anaesthetics during peripheral nerve block in rabbits. Vet Anaesth Analg. 2011;38(6):576-579.

8. Min BM, Kim JH. Epidural catheterization with a subcutaneous injection port for the long-term administration of opioids and local anesthetics to treat zoster-associated pain-a report of two cases. Korean J Anesthesiol. 2013;65(5):462-467.

9. Edlund U, Albertsson AC. Degradable polymer microspheres for controlled drug delivery. Adv Polym Sci. 2002;157:67-112.

10. Zolnik BS, Burgess DJ. Evaluation of in vivo-in vitro release of dexamethasone from PLGA microspheres. J Control Release. 2008;127(2): $137-145$.

11. Morita T, Sakamura Y, Horikiri Y, Suzuki T, Yoshino H. Evaluation of in vivo release characteristics of protein-loaded biodegradable microspheres in rats and severe combined immunodeficiency disease mice. J Control Release. 2001;73(2-3):213-221.

12. Wang $\mathrm{H}, \mathrm{Xu} \mathrm{Y}$, Zhou X. Docetaxel-loaded chitosan microspheres as a lung targeted drug delivery system: in vitro and in vivo evaluation. Int J Mol Sci. 2014;15(3):3519-3532.

13. Capan Y, Jiang G, Giovagnoli S, Na KH, Deluca PP. Preparation and characterization of poly (D, L-lactide-co glycolide) microsphere for controlled release of human growth hormone. AAPS PharmSciTech. 2003;4(2):E28.

14. Gohel MC, Amin AF. Formulation optimization of controlled release of diclofenac sodium microspheres using factorial design. J Control Release. 1998;51(2-3):115-122.

15. Artursson P, Lindmark T, Davis SS, Illum L. Effect of chitosan on the permeability of monolayers of intestinal epithelial cells (Caco-2). Pharm Res. 1994;11(9):1358-1361. 
16. Islam MA, Firdous J, Choi YJ, Yun CH, Cho CS. Design and application of chitosan microspheres as oral and nasal vaccine carriers: an updated review. Int J Nanomedicine. 2012;7:6077-6093.

17. Khare P, Jain SK. Influence of rheology of dispersion media in the preparation of polymeric microspheres through emulsification method. AAPS PharmSciTech. 2009;10(4):1295-1300.

18. Kotadiya R, Patel V, Patel H, Koradiya H. Effect of cross-linking on physicochemical properties of chitosan mucoadhesive microspheres. Int J Green Pharm. 2009;3:58-62.

19. Hassan EE, Parish RC, Gallo JM. Optimized formulation of magnetic chitosan microspheres containing the anticancer drug, oxantrazole. Pharm Res. 1992;9(3):390-397.

20. Thanoo BC, Sunny MC, Jayakrishnan A. Cross-linked chitosan microspheres: preparation and evaluation as a matrix for the controlled release of pharmaceuticals. J Pharm Pharmacol. 1992;44(4):283-286.
21. Ratajczak-Enselme M, Estebe JP, Dollo G, et al. Epidural, intrathecal and plasma pharmacokinetic study of epidural ropivacaine in PLGAmicrospheres in sheep model. Eur J Pharm Biopharm. 2009;72(1): 54-61.

22. Xu SY, Bian RL, Chen X. Methodology of Pharmacological Experiment [M]. 3rd Version. Beijing: People's Medical Publishing House; 2001: 797-889.

23. de Oliveira RM, Tanaka PP, Tenorio SB. Assessing the use of 50\% enantiomeric excess bupivacaine-loaded microspheres after sciatic nerve block in rats. Rev Bras Anestesiol. 2011;61(6):736-747.

24. Lu L, Zhang W, Wu X, et al. A novel ropivacaine-loaded in situ forming implant prolongs the effect of local analgesia in rats. Arch Med Sci. 2013;9(4):614-621.

\section{Publish your work in this journal}

Drug Design, Development and Therapy is an international, peerreviewed open-access journal that spans the spectrum of drug design and development through to clinical applications. Clinical outcomes, patient safety, and programs for the development and effective, safe, and sustained use of medicines are a feature of the journal, which has also been accepted for indexing on PubMed Central. The manuscript management system is completely online and includes a very quick and fair peer-review system, which is all easy to use. Visit http://www.dovepress.com/testimonials.php to read real quotes from published authors.

Submit your manuscript here: http://www.dovepress.com/drug-design-development-and-therapy-journal 